\title{
Artificial bioconjugates with naturally occurring linkages: the use of phosphodiester
}

\author{
Takao Shoji $^{1}$, Hiroki Fukutomi ${ }^{1}$, Yohei Okada ${ }^{2}$ and Kazuhiro Chiba ${ }^{* 1}$
}

\section{Full Research Paper}

\section{Address:}

${ }^{1}$ Department of Applied Biological Science, Tokyo University of Agriculture and Technology, 3-5-8 Saiwai-cho, Fuchu, Tokyo 183-8509, Japan and 2Department of Chemical Engineering, Tokyo University of Agriculture and Technology, 2-24-16 Naka-cho, Koganei, Tokyo 184-8588, Japan

Email:

Kazuhiro Chiba* - chiba@cc.tuat.ac.jp

* Corresponding author

Keywords:

alkyl chain soluble supports; artificial bioconjugates; naturally

occurring linkages; 5'-phosphitylation; phosphodiester bonds

\author{
Beilstein J. Org. Chem. 2018, 14, 1946-1955. \\ doi:10.3762/bjoc. 14.169 \\ Received: 18 March 2018 \\ Accepted: 06 July 2018 \\ Published: 27 July 2018 \\ Associate Editor: K. N. Allen \\ (C) 2018 Shoji et al.; licensee Beilstein-Institut. \\ License and terms: see end of document.
}

\begin{abstract}
Artificial orthogonal bond formations such as the alkyne-azide cycloaddition have enabled selective bioconjugations under mild conditions, yet naturally occurring linkages between native functional groups would be more straightforward to elaborate bioconjugates. Herein, we describe the use of a phosphodiester bond as a versatile option to access various bioconjugates. An opposite activation strategy, involving 5'-phosphitylation of the supported oligonucleotides, has allowed several biomolecules that possess an unactivated alcohol to be directly conjugated. It should be noted that there is no need to pre-install artificial functional groups and undesired and unpredictable perturbations possibly caused by bioconjugation can be minimized.
\end{abstract}

\section{Introduction}

Peptides and oligonucleotides are of exceptional importance since they are promising pharmaceutical candidates for the treatment of a range of diseases that are beyond traditional small molecule drugs [1-7]. Due to their iterative structures, chemical syntheses can technically be divided into two parts; deprotection and coupling reactions, enabling simple repeated procedures for their production, and bioactivities can poten- tially be tuned by alteration of their sequences. Nowadays, not only canonical amino acids and/or nucleosides but also artificial building blocks with various functions are available, expanding chemical libraries of peptides and oligonucleotides significantly [8-15]. Furthermore, thus designed and synthesized peptides and oligonucleotides can be conjugated with each other to integrate their bioactivities and/or functions, which has been 
one of the central topics in the field of chemical biology [1626]. Since peptide synthesis and oligonucleotide synthesis require different chemistries, such conjugations are typically carried out in the latter stages of the synthesis, otherwise subsequent steps are complicated.

Synthetic chemists, armed with various elegant artificial orthogonal bond formations, including alkyne-azide cycloaddition [27-34], thiol-ene ligation [35-38], Staudinger ligation [39,40], inverse-electron-demand Diels-Alder reaction [41-44], and hydrazone/oxime formation [45-48], have developed selective conjugation reactions under mild conditions. Although these bond-forming reactions have proven to be truly powerful approaches and will remain as first options to create novel bioconjugates, artificial functional groups such as alkynes or azides must be pre-installed into the respective biomolecules. Thus generated linkages are also artificial, possibly causing significant perturbation from their native forms, which generally affect bioactivities negatively. Arguably, naturally occurring linkages that can be formed between native functional groups would be safe alternatives, and are known as natural conjugates such as nucleopeptides and nucleolipids [49].

We have been developing alkyl chain soluble support (ACSS)assisted liquid-phase methods, specifically for peptide and oligonucleotide syntheses [50-61]. In both cases, the supported reactants and products are soluble in less-polar solvents, allowing their chemical syntheses even in submolar concentrations. The supported products are readily separated as precipitates by the addition of polar solvents, and washing the precipitates with polar solvents simultaneously rinses away excess amino acids or nucleosides and coupling reagents. We have demonstrated multistep syntheses of up to 28-mers for peptides and 21-mers for oligonucleotides without column purification. In all cases, the C-terminal-activated amino acids or 3'-terminal-activated nucleosides are coupled to the $\mathrm{N}$ - or 5 '-terminus of the supported reactants via amide or phosphodiester linkages (Figure 1). Such couplings could also be possible in the opposite activating manner. Namely, the activation of the $\mathrm{N}$ - or 5'-terminus of the supported reactants would be unique alternatives that allow the use of unactivated amino acids or nucleosides. In peptide synthesis, activation of the N-terminus is rather rare, except for some recent encouraging examples [6268]; however, this is not the case for oligonucleotide synthesis [69-73]. The activation of the 5'-primary alcohol is expected to be even more effective than that of the 3'-secondary alcohols (Figure 2), and the 5'-primary alcohol could then be coupled not only to other nucleosides but also various alcohols via a naturally occurring phosphodiester linkage. Described herein is a simple and straightforward access to artificial bioconjugates with naturally occurring linkages. (a) general peptide synthesis
activated amino acid + unactivated supported $\mathrm{N}$-terminus

Fmoc: 9-fluorenylmethyloxycarbonyl

LG: leaving group

(b) opposite peptide synthesis (limited examples)

unactivated amino acid + activated supported $\mathrm{N}$-terminus

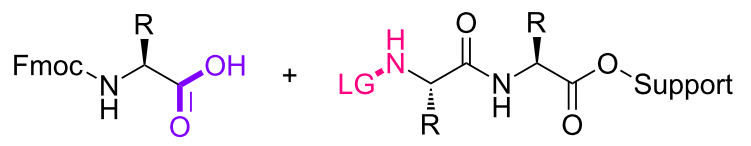

(c) general oligonucleotide synthesis

activated nucleoside + unactivated supported 5 '-terminus

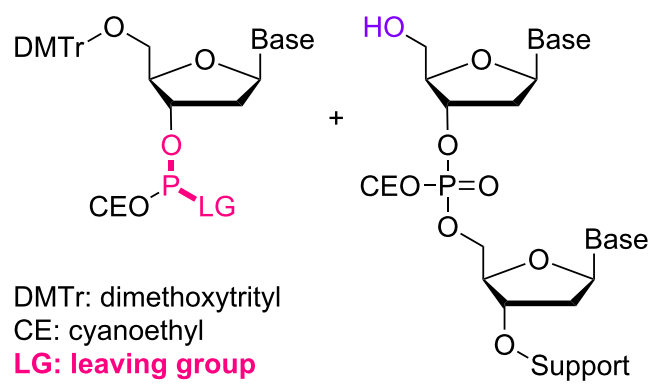

(d) opposite oligonucleotide synthesis (this work) unactivated nucleoside + activated supported 5'-terminus

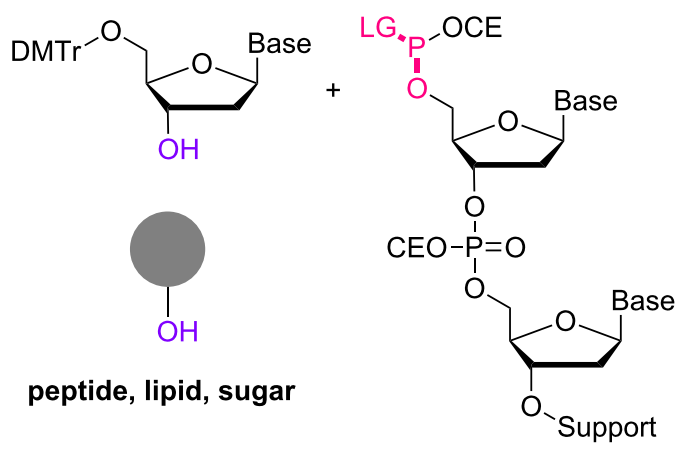

Figure 1: Schematic illustration of possible support-assisted methods.

\section{Results and Discussion}

The present work began with the optimization of the reaction conditions for the activation of the 5'-terminus, more specifically, 5'-phosphitylation [74]. The supported trinucleotide 1 was prepared from the support in $83 \%$ yield over 8 steps (Scheme S1, Supporting Information File 1) and used as a model in combination with 5-(benzylmercapto)-1 $H$-tetrazole 


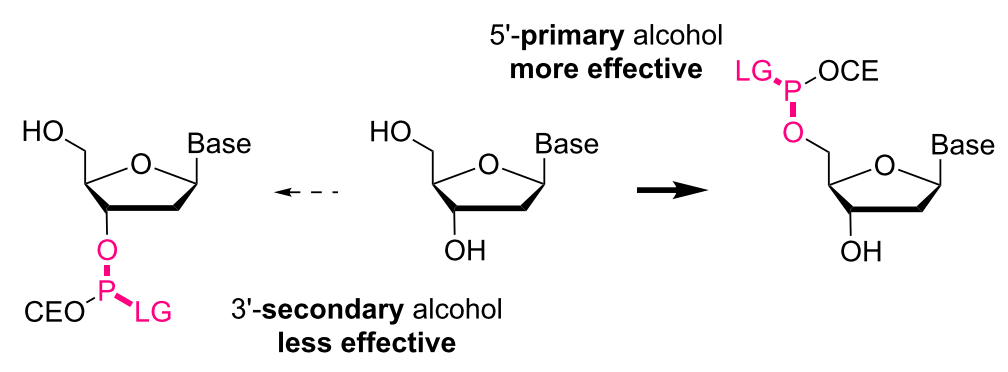

Figure 2: Expected reactivity of 5'- and 3'-terminus for the activation.

(BMT) as an activator (Table 1). The reaction was found to be rather sensitive to the concentration of the starting material (Table 1, entries 1-3). The best result was obtained at $25 \mathrm{mM}$ concentration in dichloromethane $\left(\mathrm{CH}_{2} \mathrm{Cl}_{2}\right)$. Although tetrahydrofuran (THF) is one of the typical reaction solvents for ACSS-assisted liquid-phase synthesis, this was not the case for the 5'-phosphitylation (Table 1, entry 4). When the activator was switched to tetrazole, the yield was slightly decreased
(Table 1, entry 5), while dicyanoimidazole (DCI) was proven to be an inefficient option for the reaction (Table 1, entry 6). It should be noted that the 5 '-activated supported trinucleotide 2 was stable throughout the work-up procedure routinely used for the ACSS-assisted liquid-phase method. Namely, the 5'-activated supported trinucleotide 2 was readily separated as a precipitate by the addition of acetonitrile, and washing the precipitate with acetonitrile simultaneously rinses away excess

Table 1: Optimization of the conditions for the 5'-phosphitylation.

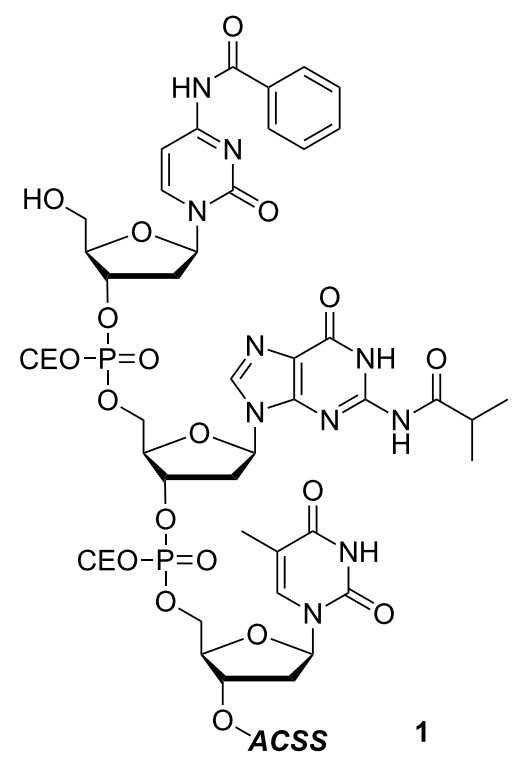

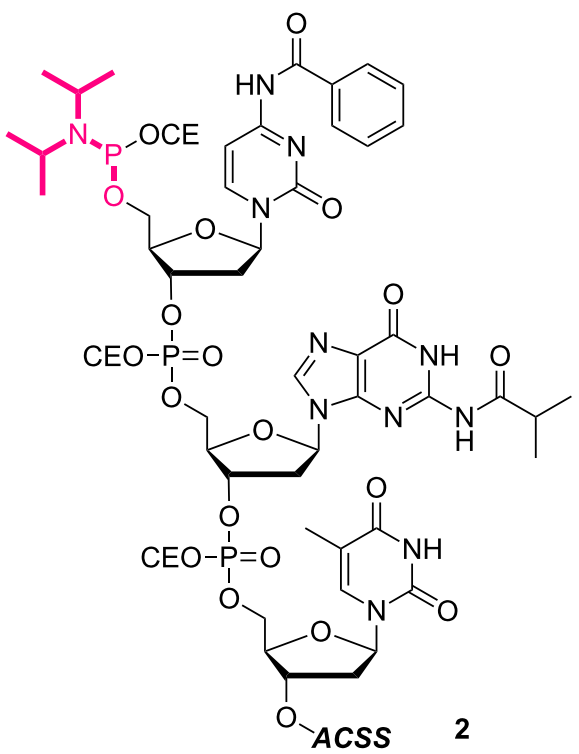

\begin{tabular}{|c|c|c|c|c|}
\hline entry $^{a}$ & activator $^{b}$ & solvent & concentration $(\mathrm{mM})$ & yield $(\%)^{\mathrm{c}, \mathrm{d}}$ \\
\hline 1 & BMT & $\mathrm{CH}_{2} \mathrm{Cl}_{2}$ & 5 & $61(24)$ \\
\hline 2 & BMT & $\mathrm{CH}_{2} \mathrm{Cl}_{2}$ & 25 & $91(0)$ \\
\hline 3 & BMT & $\mathrm{CH}_{2} \mathrm{Cl}_{2}$ & 100 & $0(81)$ \\
\hline 4 & BMT & THF & 25 & $0(86)$ \\
\hline 5 & tetrazole & $\mathrm{CH}_{2} \mathrm{Cl}_{2}$ & 25 & $83(11)$ \\
\hline 6 & $\mathrm{DCl}$ & $\mathrm{CH}_{2} \mathrm{Cl}_{2}$ & 25 & $40(46)$ \\
\hline
\end{tabular}

aReactions were carried out using 3.0 equiv of 2-cyanoethyl- $N, N, N^{\prime}, N^{\prime}$-tetraisopropylphosphorodiamidite in the presence of $3 \AA$ MS at rt for 30 min. ${ }^{b} 3.0$ equiv was used. ${ }^{C}$ Yields were determined by ${ }^{31} \mathrm{P}$ NMR analysis. ${ }^{\mathrm{d}}$ Recovered starting material was reported in parenthesis. 
reagents to afford the pure form, which could to be used for further reactions without column purification.

With the optimized 5'-phosphitylation conditions in hand, we then investigated conjugation using the 5 '-activated supported trinucleotide 2 as a model. As expected, unactivated nucleosides could be coupled to the activated 5'-terminus without difficulty (Scheme S2, Supporting Information File 1). Furthermore, to our delight, the conjugation was compatible with carboxylic acids that are potential nucleophiles for the activated 5 '-terminus and can also induce side reactions of the phosphoramidite (Scheme 1). This compatibility is of immense versatility since synthesized peptide fragments, which are cleaved from their supports thus freeing the C-terminus, could directly be conjugated to the 5 '-terminus of oligonucleotides via tyrosine, serine, or threonine side chains. In order to demonstrate such a versatile conjugation, tripeptide 3 was prepared from the support in 94\% yield over 6 steps (Scheme S3, Supporting Information File 1) and used as a model in combination with the 5 '-activated supported trinucleotide 2 (Scheme 2 and Figure 3). To our satisfaction, the conjugation took place smoothly to afford the desired bioconjugation product $\mathbf{4}$ and basic deprotection gave the native form $\mathbf{5}$ with a naturally occurring linkage in $70 \%$ yield over 5 steps. The impurities can reasonably be assigned as the hydrolized and/or oxidized products of the 5 '-activated supported trinucleotide 2 [75].

In order to investigate the generality of the current conjugation approach, we then turned our attention to the use of relatively longer peptides, sugars, and lipids as biomolecules (Table 2) [76,77]. When pentapeptide 6 was used instead (see Scheme S4 in Supporting Information File 1 for preparation of pentapeptide 6), however, the conjugation was not successful at all (Table 2, entry 1). No desired conjugate was obtained under the same reaction conditions that were effective for the conjugation with tripeptide 3 . In this case, no other functional group that could inhibit the conjugation existed in the pentapeptide $\mathbf{6}$, and therefore, it could be rationalized that the physical properties, such as solubility, were the issue. This hypothesis was confirmed by the successful conjugation with supported pentapeptide 7, affording the desired bioconjugate 8 in $67 \%$ yield over 2 steps (Table 2, entry 2). It is well-known that peptides with specific sequences can cause aggregation and thus severely inhibit further reactions; however, our support could possibly address such technical problems. Gratifyingly, although further optimization of the reaction conditions is needed for the deprotection reactions (see Schemes S5 and S6 in Supporting Information File 1 for side reactions), both the lipid 9 and the protected sugar 10 were also effectively conjugated to the activated 5'-terminus under the same reaction conditions to give the desired bioconjugates $\mathbf{1 1}$ and 12, respectively (Table 2, entries 3 and 4). We finally examined whether relatively longer supported oligonucleotides were compatible with the methodology disclosed here. Therefore, supported decanucleotide 13 was prepared from the support in 54\% yield over 27 steps (Scheme S7, Supporting Information File 1). The results were better than expected, our optimized conditions for the 5'-phosphitylation, conjugation, and deprotection were all even more effective with the supported decanucleotide $\mathbf{1 3}$ than with the supported trinucleotide $\mathbf{1}$ and the desired bioconjugate $\mathbf{1 6}$ was obtained in $84 \%$ yield over 6 steps (Scheme 3, Scheme 4 and Figure 4).

\section{Conclusion}

In conclusion, we have demonstrated that the phosphodiester bond can be an effective linkage not only to construct oligonucleotides but also to conjugate them to various biomolecules, including peptides, sugars, and lipids. The development of a method to activate the supported 5'-terminus, affording useful stable phosphoramidites that are compatible with ACSSassisted liquid-phase synthesis, has enabled direct conjugation using unactivated alcohols. It should be noted that the thus obtained bioconjugates were all artificial, but constructed via naturally occurring linkages between native functional groups. The approach presented herein should be promising to design and synthesize novel bioconjugates without undesired and unpredictable perturbations possibly derived from artificial linkages.

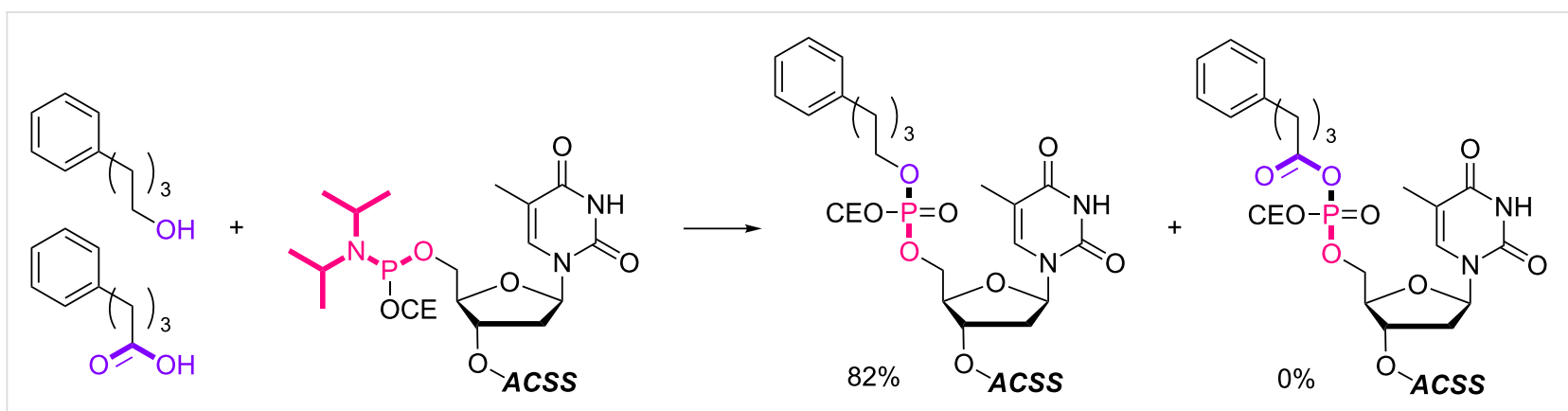

Scheme 1: Competition experiment between alcohol and carboxylic acid. Reagents and conditions: (i) 4-phenylbutyl alcohol or 4-phenylbutanoic acid (2.0 equiv), BMT (2.0 equiv), $\mathrm{CH}_{2} \mathrm{Cl}_{2} / \mathrm{CH}_{3} \mathrm{CN}$ (10:1, v/v), rt, $30 \mathrm{~min}$; (ii) $0.67 \% \mathrm{BPO} / \mathrm{DMP} / \mathrm{CH}_{2} \mathrm{Cl}_{2}, \mathrm{rt}, 30 \mathrm{~min}, 82 \%$ over 2 steps. 
<smiles>CC(C)C[C@H](NC(=O)N[C@H](CC(C)C)C(=O)N[C@H](Cc1ccc(O)cc1)C(=O)O)C(C)C</smiles>

a

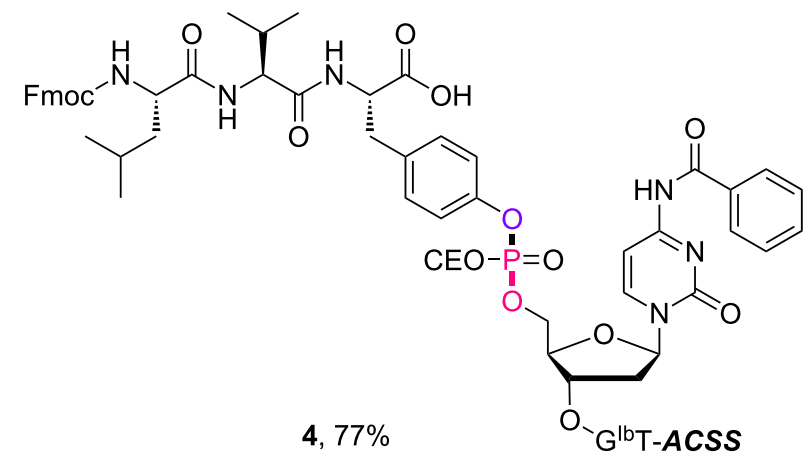

b

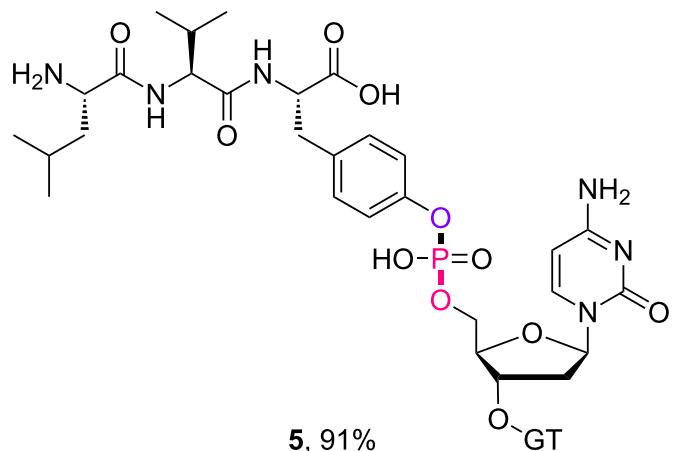

Scheme 2: Conjugation between the 5'-activated supported trinucleotide 2 and the tripeptide 3 . Reagents and conditions: (a) (i) tripeptide (2.0 equiv), BMT (2.0 equiv), $\mathrm{CH}_{2} \mathrm{Cl}_{2} / \mathrm{CH}_{3} \mathrm{CN} / \mathrm{DMF}$ (20:2:1, v/v/v), rt, $30 \mathrm{~min}$; (ii) $0.67 \% \mathrm{BPO} / \mathrm{DMP} / \mathrm{CH}_{2} \mathrm{Cl}_{2}, \mathrm{rt}, 30 \mathrm{~min}, 77 \%$ over 2 steps. (b) (i) $5 \%$ DCA/CH 5 min; (ii) $\mathrm{NH}_{3}$ aq/EtOH (3:1, v/v), $70{ }^{\circ} \mathrm{C}, 3 \mathrm{~h}$; (iii) TEA.3HF/DMF, rt, $24 \mathrm{~h}, 91 \%$ over 3 steps.
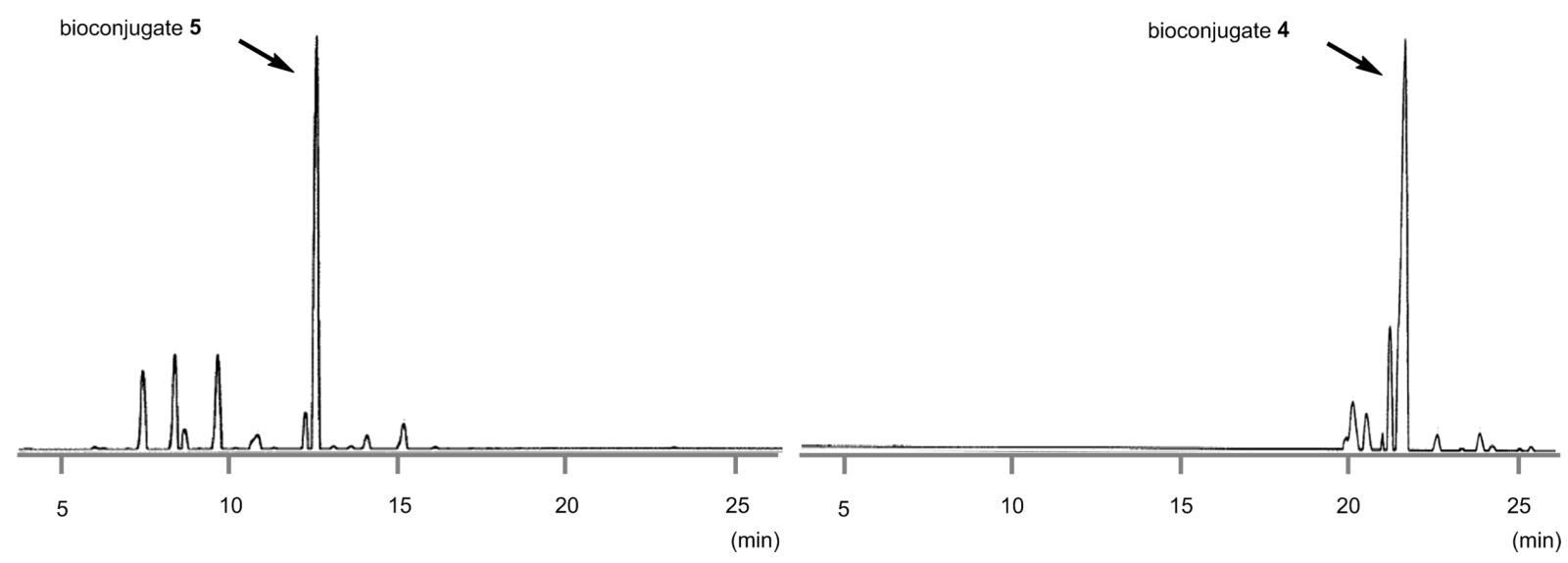

Figure 3: HPLC spectra of the crude protected bioconjugate 4 and the crude deprotected bioconjugate 5. 
Table 2: Conjugation between the 5'-activated supported trinucleotide 2 and several biomolecules. Reagents and conditions: (a) (i) biomolecule (2.0 equiv), BMT (2.0 equiv), $\mathrm{CH}_{2} \mathrm{Cl}_{2} / \mathrm{CH}_{3} \mathrm{CN} / \mathrm{DMF}$ (20:2:1, v/v/v), rt, $30 \mathrm{~min}$; (ii) $0.67 \% \mathrm{BPO} / \mathrm{DMP} \mathrm{CH}_{2} \mathrm{Cl}_{2}$, rt, $30 \mathrm{~min}$. (b) (i) $5 \% \mathrm{DCA} / \mathrm{CH} \mathrm{Cl}_{2}, \mathrm{rt}, 5 \mathrm{~min}$; (ii) $\mathrm{NH}_{3}$ aq/EtOH $(3: 1, v / v), 70^{\circ} \mathrm{C}, 3 \mathrm{~h}$; (iii) TEA 3HF/DMF, rt, $24 \mathrm{~h}$.

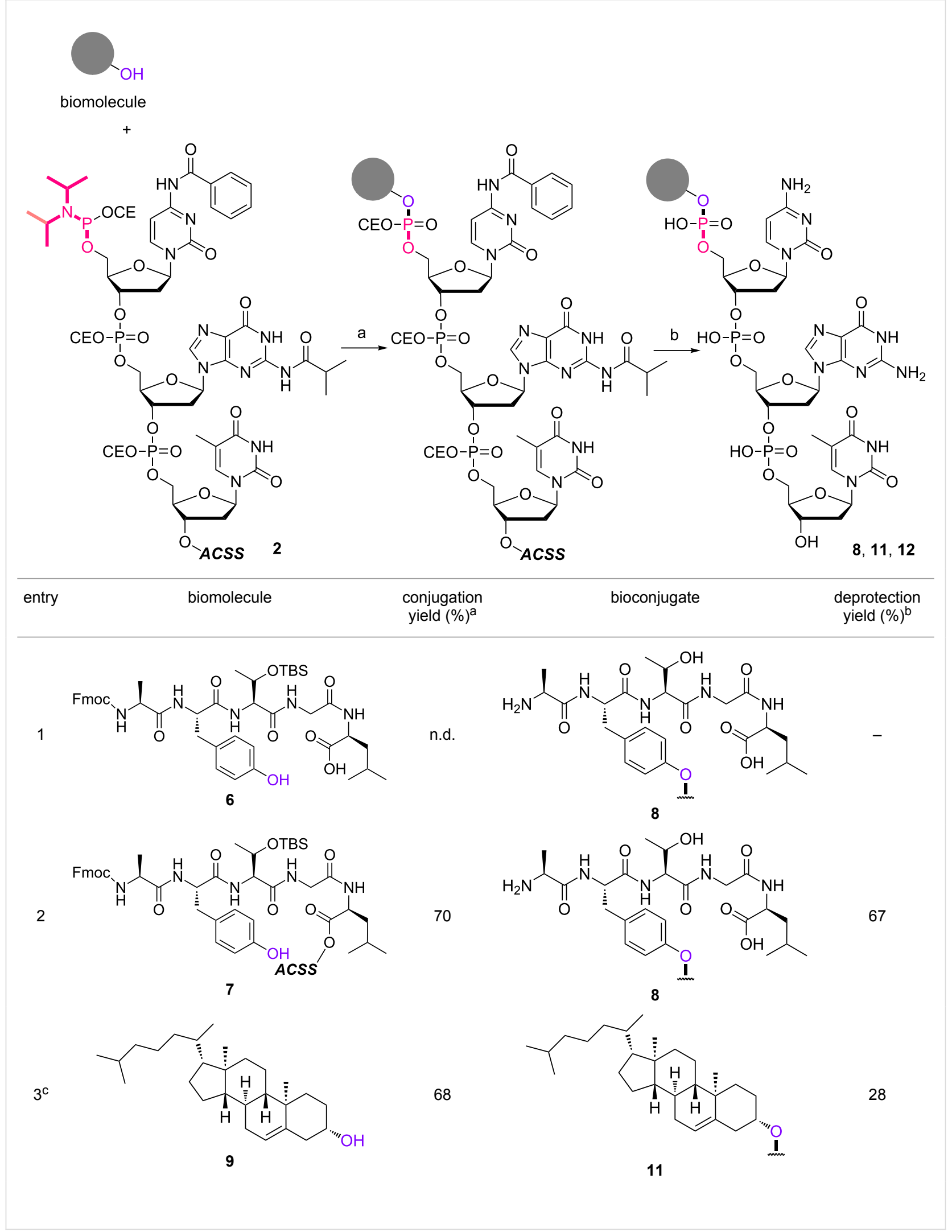


Table 2: Conjugation between the 5'-activated supported trinucleotide 2 and several biomolecules. Reagents and conditions: (a) (i) biomolecule (2.0 equiv), BMT (2.0 equiv), $\mathrm{CH}_{2} \mathrm{Cl}_{2} / \mathrm{CH}_{3} \mathrm{CN} / \mathrm{DMF}$ (20:2:1, v/v/v), rt, $30 \mathrm{~min}$; (ii) $0.67 \% \mathrm{BPO} / \mathrm{DMP} \mathrm{CH}_{2} \mathrm{Cl}_{2}$, rt, $30 \mathrm{~min}$. (b) (i) $5 \% \mathrm{DCA} / \mathrm{CH} \mathrm{Cl}_{2}, \mathrm{rt}, 5 \mathrm{~min}$; (ii) $\mathrm{NH}_{3}$ aq/EtOH $(3: 1, \mathrm{v} / \mathrm{v}), 70^{\circ} \mathrm{C}, 3 \mathrm{~h}$; (iii) TEA 3HF/DMF, rt, 24 h. (continued)

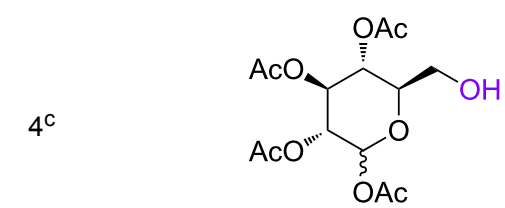

10
96<smiles>COCC1OC(O)[C@H](O)C(O)C1O</smiles>

aYields were determined by ${ }^{31} \mathrm{P}$ NMR and HPLC analyses. bYields were determined by HPLC analysis. ${ }^{c} \mathrm{CH}_{2} \mathrm{Cl}_{2} / \mathrm{CH}_{3} \mathrm{CN}(10: 1$. v/v) was used as a solvent.

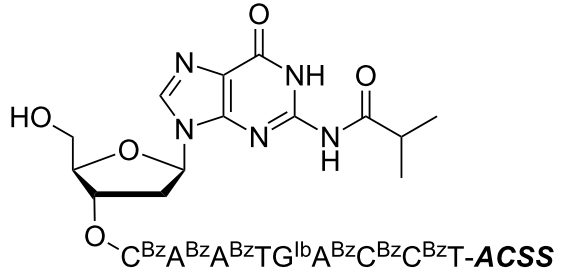

13

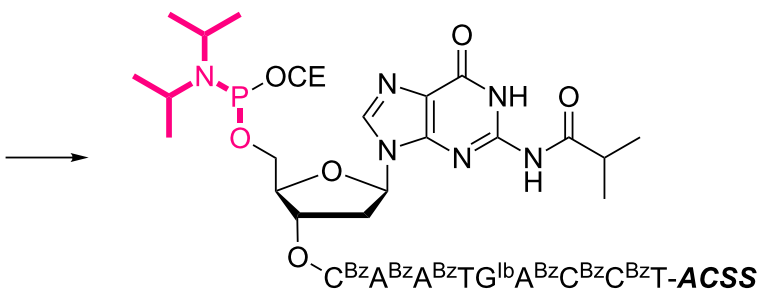

$14,88 \%$

Scheme 3: 5'-Phosphitylation of supported decanucleotide 13. Reagents and conditions: 2-cyanoethyl- $N, N, N^{\prime}, N^{\prime}$-tetraisopropylphosphorodiamidite (3.0 equiv), BMT (2.0 equiv), $\mathrm{CH}_{2} \mathrm{Cl}_{2} / \mathrm{CH}_{3} \mathrm{CN}(10: 1, \mathrm{v} / \mathrm{v}), \mathrm{rt}, 30 \mathrm{~min}, 88 \%$.

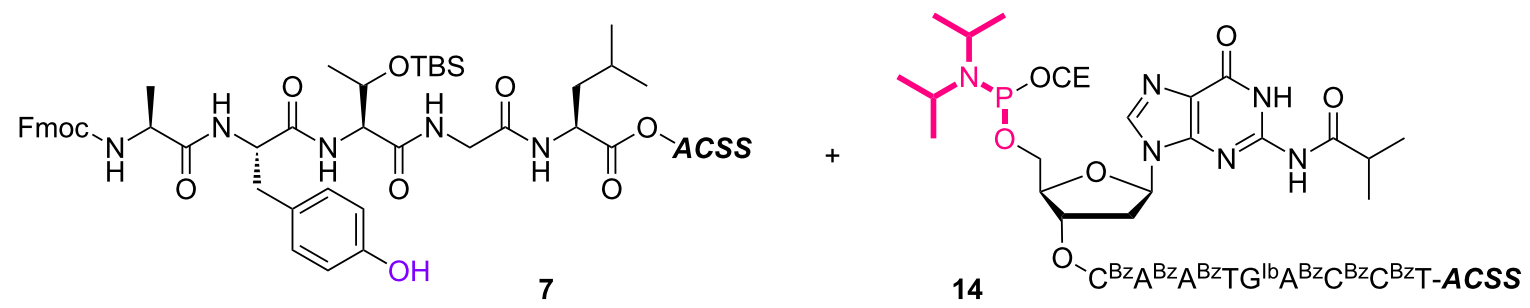

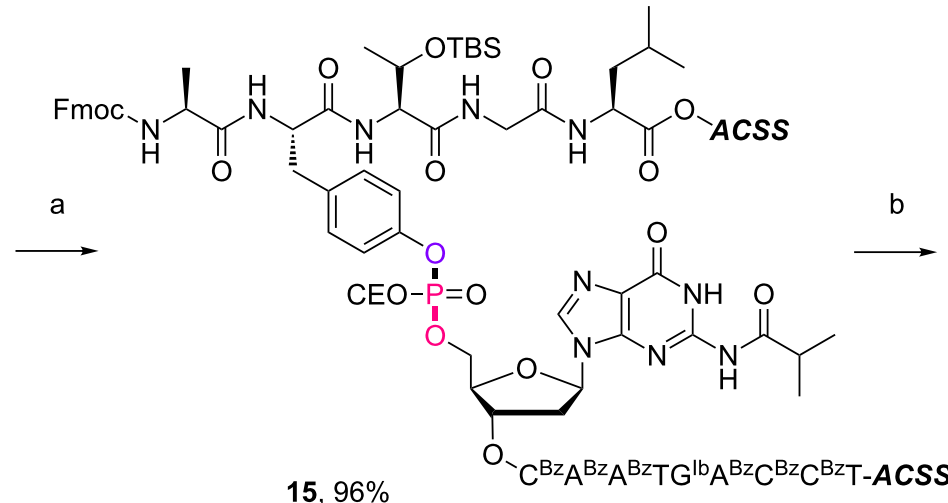

$15,96 \%$<smiles></smiles>

16, $99 \%$

Scheme 4: Conjugation between 5'-activated supported decanucleotide 14 and supported pentapeptide 7. Reagents and conditions: (a) (i) pentapeptide (2.0 equiv), BMT (2.0 equiv), $\mathrm{CH}_{2} \mathrm{Cl}_{2} / \mathrm{CH}_{3} \mathrm{CN} / \mathrm{DMF}$ (20:2:1, v/v/v), rt, $30 \mathrm{~min}$; (ii) $0.67 \% \mathrm{BPO} / \mathrm{DMP} \mathrm{CH}_{2} \mathrm{Cl}_{2}, \mathrm{rt}, 30 \mathrm{~min}, 96 \%$ over $2 \mathrm{steps}$. (b) (i) $5 \%$ $\mathrm{DCA} / \mathrm{CH}_{2} \mathrm{Cl}_{2}$, rt, 5 min; (ii) $\mathrm{NH}_{3}$ aq/EtOH $(3: 1, \mathrm{v} / \mathrm{v}), 70{ }^{\circ} \mathrm{C}, 3 \mathrm{~h}$; (iii) TEA $3 \mathrm{HF} / \mathrm{DMF}$, rt, $24 \mathrm{~h}, 99 \%$ over 3 steps. 


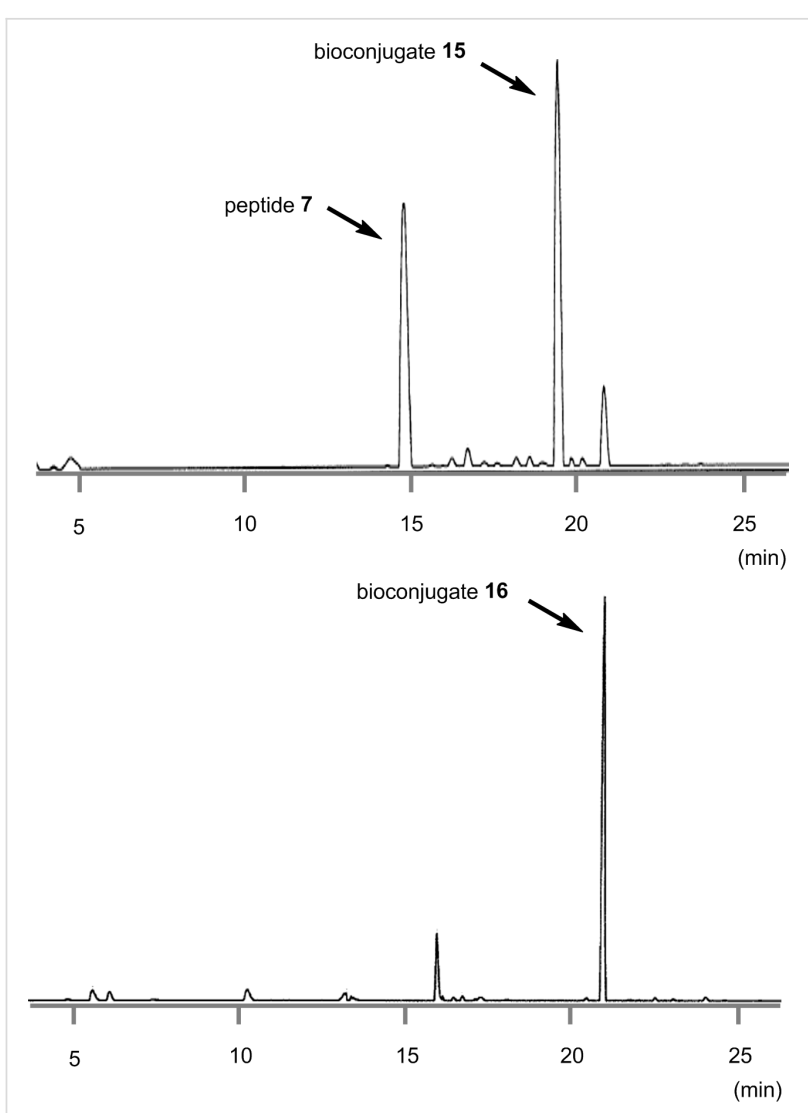

Figure 4: HPLC spectra of the crude protected bioconjugate 15 and the deprotected bioconjugate 16.

\section{Supporting Information}

\section{Supporting Information File 1}

Additional schemes and figures, general remarks, synthesis and characterization data, including copies of ${ }^{1} \mathrm{H}$ and ${ }^{13} \mathrm{C}$ NMR.

[https://www.beilstein-journals.org/bjoc/content/ supplementary/1860-5397-14-169-S1.pdf]

\section{Acknowledgements}

This work was partially supported by JSPS KAKENHI Grant Numbers 15H04494, 17K19222 (to K. C.), 16H06193, and 17K19221 (to Y. O.).

\section{ORCID ${ }^{\circledR}$ iDs}

Yohei Okada - https://orcid.org/0000-0002-4353-1595

\section{References}

1. Passioura, T.; Katoh, T.; Goto, Y.; Suga, H. Annu. Rev. Biochem. 2014, 83, 727-752. doi:10.1146/annurev-biochem-060713-035456

2. Giordanetto, F.; Kihlberg, J. J. Med. Chem. 2014, 57, 278-295. doi:10.1021/jm400887j
3. Bhat, A.; Roberts, L. R.; Dwyer, J. J. Eur. J. Med. Chem. 2015, 94, 471-479. doi:10.1016/j.ejmech.2014.07.083

4. Craik, D. J.; Fairlie, D. P.; Liras, S.; Price, D. Chem. Biol. Drug Des. 2013, 81, 136-147. doi:10.1111/cbdd.12055

5. Wittrup, A.; Lieberman, J. Nat. Rev. Genet. 2015, 16, 543-552. doi:10.1038/nrg3978

6. Mansoori, B.; Shotorbani, S. S.; Baradaran, B. Adv. Pharm. Bull. 2014, 4, 313-321. doi:10.5681/apb.2014.046

7. Khorkova, O.; Hsiao, J.; Wahlestedt, C. Pharm. Pat. Anal. 2013, 2, 215-229. doi:10.4155/ppa.13.4

8. Tao, K.; Levin, A.; Adler-Abramovich, L.; Gazit, E. Chem. Soc. Rev. 2016, 45, 3935-5679. doi:10.1039/C5CS00889A

9. Avan, I.; Hall, C. D.; Katritzky, A. R. Chem. Soc. Rev. 2014, 43, 3575-3594. doi:10.1039/c3cs60384a

10. Wojciechowskia, F.; Leumann, C. J. Chem. Soc. Rev. 2011, 40, 5669-5679. doi:10.1039/c1cs15027h

11. Bandy, T. J.; Brewer, A.; Burns, J. A.; Marth, G.; Nguyen, T.; Stulz, E. Chem. Soc. Rev. 2011, 40, 138-148. doi:10.1039/B820255A

12. Lebreton, J.; Escudier, J.-M.; Arzel, L.; Len, C. Chem. Rev. 2010, 110, 3371-3418. doi:10.1021/cr800465j

13. Sinkeldam, R. W.; Greco, N. J.; Tor, Y. Chem. Rev. 2010, 110, 2579-2619. doi:10.1021/cr900301e

14. Prakash, T. P. Chem. Biodiversity 2011, 8, 1616-1641. doi:10.1002/cbdv.201100081

15. Leumann, C. J. Bioorg. Med. Chem. 2002, 10, 841-854. doi:10.1016/S0968-0896(01)00348-0

16. Albada, B.; Metzler-Nolte, N. Chem. Rev. 2016, 116, 11797-11839. doi:10.1021/acs.chemrev.6b00166

17. Liu, S. Bioconjugate Chem. 2015, 26, 1413-1438. doi:10.1021/acs.bioconjchem.5b00327

18. Liu, H.; Irvine, D. J. Bioconjugate Chem. 2015, 26, 791-801. doi:10.1021/acs.bioconjchem.5b00103

19. Kobayashi, H.; Turkbey, B.; Watanabe, R.; Choyke, P. L. Bioconjugate Chem. 2014, 25, 2093-2100. doi:10.1021/bc500481x

20. Melnyk, O.; Ollivier, N.; Besret, S.; Melnyk, P. Bioconjugate Chem. 2014, 25, 629-639. doi:10.1021/bc500052r

21. El-Mahdi, O.; Melnyk, O. Bioconjugate Chem. 2013, 24, 735-765. doi:10.1021/bc300516f

22. Koniev, O.; Wagner, A. Chem. Soc. Rev. 2015, 44, 5495-5551. doi:10.1039/C5CS00048C

23. Singh, Y.; Murata, P.; Defrancq, E. Chem. Soc. Rev. 2010, 39 2054-2070. doi:10.1039/b911431a

24. Canalle, L. A.; Löwik, D. W. P. M.; van Hest, J. C. M. Chem. Soc. Rev. 2010, 39, 329-353. doi:10.1039/B807871H

25. Gunnoo, S. B.; Madder, A. Org. Biomol. Chem. 2016, 14, 8002-8013. doi:10.1039/C6OB00808A

26. Schilling, C. I.; Jung, N.; Biskup, M.; Schepers, U.; Bräse, S. Chem. Soc. Rev. 2011, 40, 4840-4871. doi:10.1039/c0cs00123f

27. Johansson, J. R.; Beke-Somfai, T.; Stalsmeden, A. S.; Kann, N. Chem. Rev. 2016, 116, 14726-14768. doi:10.1021/acs.chemrev.6b00466

28. Haldón, E.; Nicasio, M. C.; Pérez, P. J. Org. Biomol. Chem. 2015, 13, 9528-9550. doi:10.1039/C5OB01457C

29. Paredes, E.; Das, S. R. ChemBioChem 2011, 12, 125-131. doi:10.1002/cbic.201000466

30. El-Sagheer, A. H.; Brown, T. Chem. Soc. Rev. 2010, 39, 1388-1405. doi:10.1039/b901971p 
31. Kolb, H. C.; Finn, M. G.; Sharpless, K. B. Angew. Chem., Int. Ed. 2001, 40, 2004-2021.

doi:10.1002/1521-3773(20010601)40:11<2004::AID-ANIE2004>3.0.CO ;2-5

32. Rostovtsev, V. V.; Green, L. G.; Fokin, V. V.; Sharpless, K. B. Angew. Chem., Int. Ed. 2002, 41, 2596-2599. doi:10.1002/1521-3773(20020715)41:14<2596::AID-ANIE2596>3.0.CO ;2-4

33. Tornøe, C. W.; Christensen, C.; Meldal, M. J. Org. Chem. 2002, 67, 3057-3064. doi:10.1021/jo011148j

34. Agard, N. J.; Prescher, J. A.; Bertozzi, C. R. J. Am. Chem. Soc. 2004, 126, 15046-15047. doi:10.1021/ja044996f

35. Dondoni, A.; Marra, A. Chem. Soc. Rev. 2012, 41, 573-586. doi:10.1039/C1CS15157F

36. Hoyle, C. E.; Bowman, C. N. Angew. Chem., Int. Ed. 2010, 49, 1540-1573. doi:10.1002/anie.200903924

37. Meyer, A.; Vasseur, J.-J.; Morvan, F. Eur. J. Org. Chem. 2013, 465-473. doi:10.1002/ejoc.201201311

38. Killops, K. L.; Campos, L. M.; Hawker, C. J. J. Am. Chem. Soc. 2008, 130, 5062-5064. doi:10.1021/ja8006325

39. Weisbrod, S. H.; Baccaro, A.; Marx, A. Methods Mol. Biol. (N. Y., NY, U. S.) 2011, 751, 195-207. doi:10.1007/978-1-61779-151-2_12

40. Saxon, E.; Bertozzi, C. R. Science 2000, 287, 2007-2010. doi:10.1126/science.287.5460.2007

41. Knall, A.-C.; Slugovc, C. Chem. Soc. Rev. 2013, 42, 5131-5142. doi:10.1039/c3cs60049a

42. Bußkamp, H.; Batroff, E.; Niederwieser, A.; Abdel-Rahman, O. S.; Winter, R. F.; Wittmann, V.; Marx, A. Chem. Commun. 2014, 50, 10827-10829. doi:10.1039/C4CC04332D

43. Schoch, J.; Staudt, M.; Samanta, A.; Wiessler, M.; Jäschke, A. Bioconjugate Chem. 2012, 23, 1382-1386. doi:10.1021/bc300181n

44. Blackman, M. L.; Roysen, M.; Fox, J. M. J. Am. Chem. Soc. 2008, 130, 13518-13519. doi:10.1021/ja8053805

45. Collins, J.; Xiao, Z.; Müllner, M.; Connal, L. A. Polym. Chem. 2016, 7, 3812-3826. doi:10.1039/C6PY00635C

46. Meyer, A.; Spinelli, N.; Dumy, P.; Vasseur, J. J.; Morvan, F.; Defrancq, E. J. Org. Chem. 2010, 75, 3927-3930. doi:10.1021/jo100599m

47. Dirksen, A.; Hackeng, T. M.; Dawson, P. E. Angew. Chem., Int. Ed. 2006, 45, 7581-7584. doi:10.1002/anie.200602877

48. Zatsepin, T. S.; Stetsenko, D. A.; Arzumanov, A. A.; Romanova, E. A.; Gait, M. J.; Oretskaya, T. S. Bioconjugate Chem. 2002, 13, 822-830. doi:10.1021/bc020016+

49. Rosemeyer, H. Chem. Biodiversity 2005, 2, 977-1063. doi:10.1002/cbdv.200590082

50. Ogami, K.; Okada, Y.; Chiba, K. Chem. Lett. 2018, 47, 138-140. doi:10.1246/cl.170971

51. Wakamatsu, H.; Okada, Y.; Sugai, M.; Hussaini, S. R.; Chiba, K. Asian J. Org. Chem. 2017, 6, 1584-1588. doi:10.1002/ajoc.201700401

52. Okada, Y.; Asama, H.; Wakamatsu, H.; Chiba, K.; Kamiya, H. Eur. J. Org. Chem. 2017, 5961-5965. doi:10.1002/ejoc.201700697

53. Matsuno, Y.; Shoji, T.; Kim, S.; Chiba, K. Org. Lett. 2016, 18, 800-803. doi:10.1021/acs.orglett.6b00077

54. Okada, Y.; Wakamatsu, H.; Sugai, M.; Kauppinen, E. I.; Chiba, K. Org. Lett. 2015, 17, 4264-4267. doi:10.1021/acs.orglett.5b02057

55. Okada, Y.; Hosoya, S.; Suzuki, H.; Chiba, K. Org. Lett. 2014, 16, 6448-6451. doi:10.1021/ol5032798

56. Shoji, T.; Kim, S.; Chiba, K. Chem. Lett. 2014, 43, 1251-1253. doi:10.1246/cl.140355
57. Kitada, S.; Fujita, S.; Okada, Y.; Kim, S.; Chiba, K. Tetrahedron 2013, 69, 2555-2559. doi:10.1016/j.tet.2013.01.068

58. Okada, Y.; Suzuki, H.; Nakae, T.; Fujita, S.; Abe, H.; Nagano, K.; Yamada, T.; Ebata, N.; Kim, S.; Chiba, K. J. Org. Chem. 2013, 78, 320-327. doi:10.1021/jo302127d

59. Fujita, Y.; Fujita, S.; Okada, Y.; Chiba, K. Org. Lett. 2013, 15, 1155-1157. doi:10.1021/ol4003477

60. Kitada, S.; Takahashi, M.; Yamaguchi, Y.; Okada, Y.; Chiba, K. Org. Lett. 2012, 14, 5960-5963. doi:10.1021/ol302863r

61. Tana, G.; Kitada, S.; Fujita, S.; Okada, Y.; Kim, S.; Chiba, K. Chem. Commun. 2010, 46, 8219-8221. doi:10.1039/c0cc03090b

62. Zhu, Y.-P.; Sergeyev, S.; Franck, P.; Orru, R. V. A.; Maes, B. U. W. Org. Lett. 2016, 18, 4602-4605. doi:10.1021/acs.orglett.6b02247

63. Wu, X.; Stockdill, J. L.; Park, P. K.; Danishefsky, S. J. J. Am. Chem. Soc. 2012, 134, 2378-2384. doi:10.1021/ja2103372

64. Li, X.; Yuan, Y.; Kan, C.; Danishefsky, S. J. J. Am. Chem. Soc. 2008, 130, 13225-13227. doi:10.1021/ja804709s

65. Suppo, J.-S.; Subra, G.; Berges, M.; de Figueiredo, R. M.; Campagne, J.-M. Angew. Chem., Int. Ed. 2014, 53, 5389-5393. doi:10.1002/anie.201402147

66. Grzyb, J. A.; Batey, R. A. Tetrahedron Lett. 2003, 44, 7485-7488. doi:10.1016/j.tetlet.2003.08.026

67. Kosal, A. D.; Wilson, E. E.; Ashfeld, B. L. Angew. Chem., Int. Ed. 2012, 51, 12036-12040. doi:10.1002/anie.201206533

68. Hegarty, A. F.; McCormack, M. T.; Ferguson, G.; Roberts, P. J. J. Am. Chem. Soc. 1977, 99, 2015-2016. doi:10.1021/ja00448a075

69. van der Heden van Noort, G. J.; van Delft, P.; Meeuwenoord, N. J.; Overkleeft, H. S.; van der Marel, G. A.; Filippov, D. V. Chem. Commun. 2012, 48, 8093-8095. doi:10.1039/c2cc33477a

70. van der Heden van Noort, G. J.; Overkleeft, H. S.; van der Marel, G. A.; Filippov, D. V. J. Org. Chem. 2010, 75, 5733-5736. doi:10.1021/jo100757t

71. Kriek, N. M. A. J.; Meeuwenoord, N. J.; van den Elst, H.; Heus, H. A.; van der Marel, G. A.; Filippov, D. V. Org. Biomol. Chem. 2006, 4, 3576-3586. doi:10.1039/b608544j

72. Schmidt, K. S.; Filippov, D. V.; Meeuwenoord, N. J.; van der Marel, G. A.; van Boom, J. H.; Lippert, B.; Reedijk, J. Angew. Chem., Int. Ed. 2000, 39, 375-377. doi:10.1002/(SICI)1521-3773(20000117)39:2<375::AID-ANIE375>3.0. CO;2-M

73. Zhou, X.; Remaud, G.; Chattopadhyaya, J. Tetrahedron 1988, 44, 6471-6489. doi:10.1016/S0040-4020(01)89837-9

74. Wei, X. Tetrahedron 2013, 69, 3615-3637. doi:10.1016/j.tet.2013.03.001

75. Kungurtsev, V.; Laakkonen, J.; Molina, A. G.; Virta, P. Eur. J. Org. Chem. 2013, 6687-6693. doi:10.1002/ejoc.201300864

76. Venkatesan, N.; Kim, B. H. Chem. Rev. 2006, 106, 3712-3761. doi:10.1021/cr0502448

77. Spinelli, N.; Defrancq, E.; Morvan, F. Chem. Soc. Rev. 2013, 42, 4557-4573. doi:10.1039/C2CS35406C 


\section{License and Terms}

This is an Open Access article under the terms of the Creative Commons Attribution License

(http://creativecommons.org/licenses/by/4.0). Please note that the reuse, redistribution and reproduction in particular requires that the authors and source are credited.

The license is subject to the Beilstein Journal of Organic Chemistry terms and conditions:

(https://www.beilstein-journals.org/bjoc)

The definitive version of this article is the electronic one which can be found at:

doi:10.3762/bjoc. 14.169 